\title{
AUTHOR INDEX \\ Volume 21
}

Akl, S. G., Editorial note

Akl, S. G., Editorial note

Akl, S. G., Editorial note

Akl, S. G., Twentieth anniversary issue: Editorial note

Alexandrov, V., see Varghese, B.

Alves, M. A. Z., Freitas, H. C. and Navaux, P. O. A., High latency and contention on shared L2-cache for many-core architectures

Ayguadé, E., see Duran, A.

Badia, R. M., see Duran, A.

Balaji, P., Buntinas, D., Goodell, D., Gropp, W., Hoefler, T., Kumar, S., Lusk, E., Thakur, R. and Träff, J. L., MPI on millions of cores

Banu, N., Izumi, T. and Wada, K., Adaptive and doubly-expedited one-step consensus in byzantine asynchronous systems

Barker, K. J., see Kerbyson, D. J.

Buntinas, D., see Balaji, P.

Cappello, F., Casanova, H. and Robert, Y., Preventive migration vs. preventive checkpointing for extreme scale supercomputers

Casanova, H., see Cappello, F.

Ceres, P. and Ceres, R., On the fault-tolerance and size of WDM optical networks

Ceres, R., see Ceres, P.

Chen, S.-D., see Lee, C.-C.

Cheng, E., Qiu, K. and Shen, Z., On the surface areas and average distances of meshes and tori

Chung, I.-H., Lee, C.-R., Zhou, J. and Chung, Y.-C., Hierarchical mapping for HPC applications

Chung, Y.-C., see Chung, I.-H.

Clarke, D., Lastovetsky, A. and Rychkov, V., Dynamic load balancing of parallel computational iterative routines on highly heterogeneous HPC platforms

Diakité, S., Marchal, L., Nicod, J.-M. and Philippe, L., Practical steady-state scheduling for tree-shaped task graphs

Dongarra, J. and Tourancheau, B., Guest editors note on clusters, clouds and grids for scientific computing
21 (2011) 1

21 (2011) 107

21 (2011) 377

21 (2011) 273

21 (2011) 379-396

21 (2011) 85-106

21 (2011) 173-193

21 (2011) 173-193

21 (2011) 45-60

21 (2011) 461-477

21 (2011) 301-318

21 (2011) 45-60

21 (2011) 111-132

21 (2011) 111-132

21 (2011) 3-12

21 (2011) 3-12

21 (2011) 21-26

21 (2011) 61-75

21 (2011) 279-299

21 (2011) 279-299

21 (2011) 195-217

21 (2011) 397-412

21 (2011) 109 
Duran, A., Ayguadé, E., Badia, R. M., Labarta, J., Martinell, L., Martorell, X. and Planas, J., OmpSs: A proposal for programming heterogeneous multi-core architectures

21 (2011) 173-193

Emmart, N. and Weems, C. C., High precision integer multiplication with a GPU using Strassen's algorithm with multiple FFT sizes

21 (2011) 359-375

Fehske, H., see Schubert, G.

Freitas, H. C., see Alves, M. A. Z.

Gamboa, C. F. and Robertazzi, T., Simple performance bounds for multicore and parallel channel systems

Goodell, D., see Balaji, P.

Grimshaw, A., see Merrill, D.

Gropp, W., see Balaji, P.

Hager, G., see Schubert, G.

Hoefler, T., see Balaji, P.

Hwang, M.-S., see Tzeng, S.-F.

Izumi, T., see Banu, N.

Jesshope, C. R., see Uddin, M. I.

Jetley, P., see Sun, Y.

Kale, L. V., see Sun, Y.

Kerbyson, D. J. and Barker, K. J., Modeling the performance of direct numerical simulation on parallel systems

Kerbyson, D. J., see Weems, C. C.

Kielmann, T., see Oprescu, A.-M.

Kumar, S., see Balaji, P.

Labarta, J., see Duran, A.

Lastovetsky, A., see Clarke, D.

Leahu, H., see Oprescu, A.-M.

Lee, C.-C., Li, C.-T. and Chen, S.-D., Two attacks on a two-factor user authentication in wireless sensor networks

21 (2011) 339-358

21 (2011) 85-106

21 (2011) 439-460

21 (2011) 45-60

21 (2011) 245-272

21 (2011) 45-60

21 (2011) 339-358

21 (2011) 45-60

21 (2011) 77-84

21 (2011) 461-477

21 (2011) 413-438

21 (2011) 319-338

21 (2011) 319-338

21 (2011) 301-318

21 (2011) 275-277

21 (2011) 219-243

21 (2011) 45-60

21 (2011) 173-193

21 (2011) 195-217

21 (2011) 219-243

Lee, C.-C., see Tzeng, S.-F.

Lee, C.-R., see Chung, I.-H.

Lefevre, L., see Orgerie, A.-C.

Li, C.-T., see Lee, C.-C.

Lin, S.-S., see Pan, T.-T.

Lu, D., see Walker, C.

Lusk, E., see Balaji, P.

Marchal, L., see Diakité, S.

Martinell, L., see Duran, A.

21 (2011) 21-26

21 (2011) 77-84

21 (2011) 279-299

21 (2011) 133-154

21 (2011) 21-26

21 (2011) 27-43

21 (2011) 155-171

21 (2011) 45-60

21 (2011) 397-412

21 (2011) 173-193

21 (2011) 173-193

Martorell, X., see Duran, A.

21 (2011) 379-396

Merrill, D. and Grimshaw, A., High performance and scalable radix sorting: A case study of implementing dynamic parallelism for GPU computing

21 (2011) 245-272

Navaux, P. O. A., see Alves, M. A. Z.

21 (2011) 85-106

Nicod, J.-M., see Diakité, S.

21 (2011) 397-412 
Oprescu, A.-M., Kielmann, T. and Leahu, H., Budget estimation and control for bag-of-tasks scheduling in clouds

21 (2011) 219-243

Orgerie, A.-C. and Lefevre, L., ERIDIS: Energy-efficient reservation infrastructure for large-scale distributed systems

Pan, T.-T. and Lin, S.-S., The transitive closure and related algorithms of digraph on the reconfigurable architecture

21 (2011) 133-154

21 (2011) 27-43

Philippe, L., see Diakité, S.

21 (2011) 397-412

Planas, J., see Duran, A.

21 (2011) 173-193

Qiu, K., see Cheng, E.

Rajamony, R., see Weems, C. C.

21 (2011) 61-75

21 (2011) 275-277

Robert, Y., see Cappello, F.

Robertazzi, T. G., A product form solution for tree networks with divisible loads

21 (2011) 111-132

21 (2011) 13-20

21 (2011) 439-460

Robertazzi, T., see Gamboa, C. F.

21 (2011) 195-217

Schubert, G., Fehske, H., Hager, G. and Wellein, G.,

Hybrid-parallel sparse matrix-vector multiplication with explicit communication overlap on current multicore-based systems

Shen, Z., see Cheng, E.

Sun, Y., Zheng, G., Jetley, P. and Kale, L. V., ParSSSE: An adaptive parallel state space search engine

Thakur, R., see Balaji, P.

Tourancheau, B., see Dongarra, J.

Träff, J. L., see Balaji, P.

Tzeng, S.-F., Lee, C.-C. and Hwang, M.-S., A batch verification for multiple proxy signature

Uddin, M. I., Van Tol, M. W. and Jesshope, C. R., High level simulation of SVP many-core systems

Van Tol, M. W., see Uddin, M. I.

Varghese, B., McKee, G. and Alexandrov, V., Can agent intelligence be used to achieve fault tolerant parallel computing systems?

21 (2011) 379-396

21 (2011) 461-477

Wada, K., see Banu, N.

Walker, C., Lu, D. and Walker, D. W., Automatic portal generation based on workflow description

21 (2011) 155-171

Walker, D. W., see Walker, C.

Weems, C. C., Kerbyson, D. J. and Rajamony, R., Guest editor's note: Large-scale parallel processing

21 (2011) 339-358

21 (2011) 61-75

21 (2011) 319-338

21 (2011) 45-60

21 (2011) 109

21 (2011) 45-60

21 (2011) 77-84

21 (2011) 413-438

21 (2011) 413-438

21 (2011) 155-171

21 (2011) 275-277

21 (2011) 359-375

21 (2011) 339-358

Wellein, G., see Schubert, G.

21 (2011) 319-338

Zheng, G., see Sun, Y.

21 (2011) 279-299

Zhou, J., see Chung, I.-H. 